\title{
IoT based Employee Tracking and Management System
}

\author{
Suvarna Patil \\ Faculty, Computer Engineering \\ DYPIEMR, Pune
}

\author{
Nikhil Agarwal \\ Student, Computer Engineering \\ DYPIEMR, Pune
}

\author{
Rupak Choudhary \\ Student, Computer Engineering \\ DYPIEMR, Pune
}

\author{
Sujeet Dande \\ Student, Computer Engineering \\ DYPIEMR, Pune
}

\author{
Rishi Raj \\ Student, Computer Engineering \\ DYPIEMR, Pune
}

\begin{abstract}
IoT is simply the interconnection of things through the power of the internet. Internet connected devices used built-in sensors to collect the data and in some cases act on it. In this paper, we have discussed about two systems- Counting System and the Code Scanner System which are an integration of basic electronics components and technology and can prove to be very essential in certain applications. The counting system can help in maintaining live count of number of objects or people in a certain scenario, the code scanner system can be used to uniquely identify objects or people for the machine and perform operations accordingly. These systems can be used for other applications too by just making slight changes. The paper talks about a few of such application where these systems can be implemented and brought to power.
\end{abstract}

\section{General Terms}

Attendance, Home Automation

\section{Keywords}

Internet of Things, Counter, Attendance System

\section{INTRODUCTION}

Internet of Things (IoT) has transformed tremendously over the past decade. People have moved from traditional manual methods to automating almost every small task in life. IoT has come up as the bridge between the past and the future with advancements in all sectors be it from agriculture to multinational companies. IoT has extensively helped in the controlling, monitoring and automating daily repetitive tasks which human initially did all by themselves. IoT has the potential to connect objects with each other and the surrounding with the help of the internet. The passionate technocrats worked their way to automate tasks from houses to industries with the power of the internet of things. IoT provides a platform to integrate multiple small components and build a system to solve to solve a thriving problem. In industries, hundreds of employees are required to keep track of small basic repetitive aspects. IoT cuts off, the requirement of such management by automating just basic processes and providing speedy results at the power of a click. ERP systems are one aspect wherein IoT is on the rise to automate the entire structure and ease out the entire process.

Bringing IoT technology to be implemented in organizations can enhance efficiency, save time, improve accuracy, provide advanced security and most important can help to control the system on the go with the help of mobile phones and laptops. It is said that almost 30 billion devices would be connected to the
Internet of Things by the end of 2020 with the number touching almost 75 billion by 2025 .

\begin{abstract}
Various small components coupled with the technology can help to make powerful systems to solve human problems. A single system can be used in various fields for multiple different applications by just tweaking the end node of the results. In this paper, we talk about two such systems which is a minimal integration of components and technologies but can prove to be very crucial when put to use. The paper talks about a counting system which can help to keep live track of objects/people and can send data over the internet to serve to the authority through a mobile application. Another system mentioned in the paper is about a system which can help in monitoring and tracking objects as well as can be tweaked to use for attendance management in organizations.
\end{abstract}

\section{RELATED WORK}

The Smart Attendance System proposed by Qianwen Miao, Fu Xiao , Haiping Huang, Lijuan Sun, and Ruchuan Wang proposes a device-free attendance system for offices with respect to data collected from RFID reader. This paper refers to the use of a frequency distribution histogram and a $\mathrm{K}$ means algorithm to improve the systems accuracy. The average accuracy achieved from this research is $92 \%$. [1].

Educational Institutions have been looking at opportunities to improve the educational environment by using cutting-edge technology. This paper by Masalha, F., \& Hirzallah, N. proposes a model to use mobile technology to effectively benefit from the entire time allotted to a lecture. Time spent by instructors taking attendance can be seen as a waste of lecture time, particularly when classes are large. This paper suggests a method to simplify the procedure by using the students' devices instead of instructor's device. [2].

This research paper, by Khoenkaw, P. and Pramokchon, P., describes a portable bi-directional automated people counting system. The proposed approach is split into two parts: hardware and signal processing algorithms. A single ultrasonic range finder module is used to perform the hardware component. The signal processing section employs a data fitting system to classify the individual and walking path from the sensor signal. [3]

The studies performed in this paper shows that classroom attendance is recorded at the cost of teaching learning time. As a result, the current paper aims to explore an appropriate method of recording attendance that does not interfere with learning. This paper introduces and addresses a smart 
attendance management device in a smart class room as a way of reducing dropout rates by using linked items and RFID technologies. This device will allow the montitoring of students' attendance at present using IoT while sending attendance records to administration. At the same time, parents will track their children's attendance in class by receiving an email or SMS message. Furthermore, the device automatically sends missing lessons to students that are absent. [4].

The smart card is a thriving technology. The use of smart cards would increase overall reliability, productivity due to a cashless world, data accuracy and student card accessibility. Many changes to the current environment can be made using the flexible smart card applications. Education is not only one area where smart cards can be used; smart cards can be used in a variety of industries, and using them improves their functionality, efficiency and usability. [6].

\section{USE CASES \\ 3.1. Counting System}

A counting system is an application of IoT for which can be used to keep track of the quantity of objects passing through a gateway. This can be used to keep track of things on a conveyer belt or track of number of people in a room at a given time, etc. The flow of the system would be in such a way that we will have sensors connected at the gateway from which the object is entering/exiting. This sensor would sense the passing of the object and send signal to the controller. The controller would perform the logical operation in accordance with the signal and provide the output to the display or actuator. The display can then show the count or if using an actuator, it can perform the task set of a particular count, for e.g. stopping the conveyor belt if the count of objects reaches a set threshold. The same data can also be sent over the internet to a mobile application which will be accessed by the concerned authority on the go.

- Sensors: We can use any from the variety of distance sensors available. The task of the sensor majorly is to detect a passing object and send signal to the controller. Examples of sensors that can be used are Ultrasonic sensors, IR sensors, PIR sensors, etc.

- Controller: The controller is the brain of the entire system. Controllers have the potential to perform certain logical operations and receive and transmit signals. The controller can be any controller available like Arduino, NodeMCU, Raspberry Pi, etc.

- Display/Actuator: The actuators or display would be the output device of the system. The display can be used to display the live count based on the signal received from the sensor to the controller. An actuator can be used if needed to perform a certain operation upon reaching a certain count.

- Mobile Application: The real time data would be sent to the application and the authority concerned will be able to view this data from anywhere in the world.

\subsection{Code Scanner System for Tracking}

Nowadays, various types of data encrypted code are available to save data like QR Codes, Barcodes, RFID Tags, etc. These codes can be used to store small amount of data. Every code or tag would be unique and can be used as a unique id. This code can then anytime be retrieved using the corresponding code reader. The system can be then used in many different ways and have diverse applications. The flow of the system would be this way that the user/object has a unique code which it will flash in front of the reader. The reader would read the code and translate it into data. This would be sent to the controller. The controller would, based on the logic applied, perform the operation. The output can also be seen on the go with the help of a mobile application too.

- Code/Tag: A code is a machine readable image which can be read to retrieve data. The code is the unique identifier which helps in differentiating between objects and to uniquely identify a particular entity. Data of the entity would be stored on the tag/code. Examples of codes can be QR codes, Barcodes, RFID tags, etc.

- Scanner/Reader: A scanner/reader is a tool which captures data from the tag and converts it to human readable data. Examples of scanners can be QR code scanner, barcode reader, RFID reader, etc.

- Controller: The controller is the brain of the entire system. Controllers have the potential to perform certain logical operations and receive and transmit signals. The controller can be any controller available like Arduino, NodeMCU, Raspberry Pi, etc.

- Display/Actuator: The actuators or display would be the output device of the system. The display can be used to display the data received from the reader to the controller. An actuator can be used if needed to perform a certain operation upon reading a particular data.

- Mobile Application: The real time data would be sent to the application and the authority concerned will be able to view this data from anywhere in the world. This data can also be stored for future use.

\section{SYSTEM ARCHITECTURE}

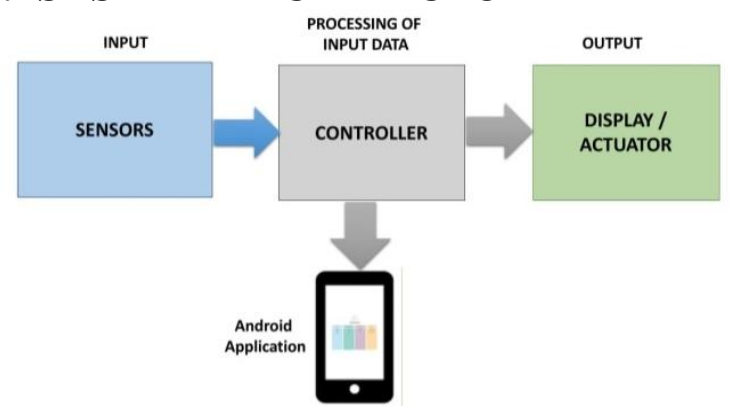

Fig 1: Block Diagram - Counting System

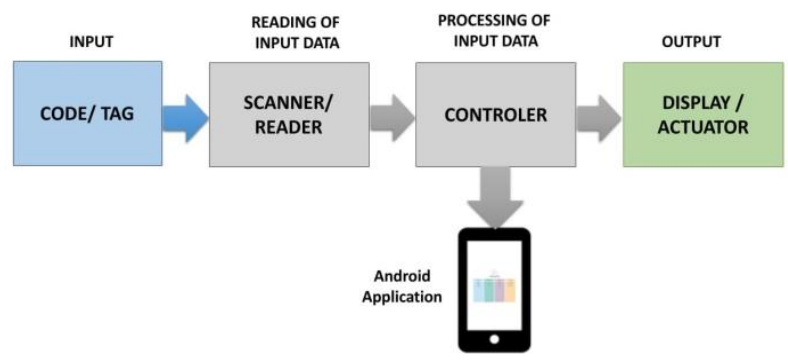

Fig 2: Block Diagram - Code Scanner System 


\section{APPLICATIONS}

\subsection{Counting System}

- Malls: These systems can be setup near the entrances of malls to have track of the step-ins in a day. This can be used for analysis for the mall.

- Manufacturing Line: Counting system could be setup on the manufacturing lines to have track of the count of number of objects being manufactured

- Multination companies: This system can be used to keep live track of number of employees in a room and on a floor.

- Educational Institutions: This system can be used to keep live track of number of students entering the school as well as to keep track of the number of students in any given classroom.

- Auditoriums/ Cinema Halls: This can be used to know the number of people entered and eventually to find out the number of available seats in the auditorium/cinema hall.

\subsection{Code Scanner System}

- Attendance Management System: Every employee can have a unique code which can be used to mark his day to day attendance.

- Object Tracking System: This system can be used in malls or stores to keep track of objects and uniquely identify them.

- Library: Every book can have a code on it which can help to identify its location in the library. Ever users can have unique ids on the library cards to help retrieve their data on the computer systems.

- $\quad$ Fast Tags: Tags on vehicles can be used to detect if the vehicle is allowed in the campus and open the entrance gate upon authentication.

- Home Automation: QR codes or RFIDs can be used to lock and unlock the main door of a house and similarly for cupboards

\section{CONCLUSION}

IoT can prove to be a great technology for integrating virtual world with the physical world. Connected systems can work in unison to provide high end technology with simplicity. Systems like counting system and code scanner systems can be used in various applications independent of the sector. These systems are easy to implement and powerful to be used. In future, these can be integrated with various other technologies to be used on a large scale.

\section{REFERENCES}

[1] Miao, Q., Xiao, F., Huang, H., Sun, L., \& Wang, R. (2019). Smart attendance system based on frequency distribution algorithm with passive RFID tags. Tsinghua Science and Technology, 25(2), 217-226.

[2] Masalha, F., \& Hirzallah, N. (2014). A students attendance system using QR code. International Journal of Advanced Computer Science and Applications, 5(3), 75-79.

[3] Khoenkaw, P., \& Pramokchon, P. (2020, March). Bidirectional Portable Automatic People Counting Using A
Single Ultrasonic Range Finder. In 2020 Joint International Conference on Digital Arts, Media and Technology with ECTI Northern Section Conference on Electrical, Electronics, Computer and Telecommunications Engineering (ECTI DAMT \& NCON) (pp. 34-37). IEEE.

[4] El Mrabet, H., \& Moussa, A. A. (2020). IoT-School Attendance System Using RFID Technology.

[5] Malathy, E. M., Sivamurugan, V., \& Rajtilak, S. (2020, September). Performance Analysis and Enhancement to Biometric based Attendance System. In 2020 4th International Conference on Computer, Communication and Signal Processing (ICCCSP) (pp. 1-3). IEEE.

[6] Jesani, N., Gupta, N., Bhatt, S., Singh, P., \& Saxena, A. (2020, February). Smart Card For Various Application In Institution. In 2020 IEEE International Students' Conference on Electrical, Electronics and Computer Science (SCEECS) (pp. 1-5). IEEE

[7] Panca, B. S., Paulus, Y., \& Karnalim, O. (2020). An Automated Attendance System in Academia with Implicit Authentication via Mobile Devices and $\mathrm{Wi}-\mathrm{Fi}$ Connection. International Journal of Online \& Biomedical Engineering, 16(7).

[8] Hendry, R., Rahman, M. N. A., \& Seyal, A. H. (2017). Smart attendance system applying QR code. In Proceedings of the 12th International Conference on Latest Trends in Engineering and Technology (ICLTET'2017); the 7th International Conference on Agricultural, Chemical, Biological and Environmental Sciences (ACBES'2017).

[9] Wei, X., Manori, A., Devnath, N., Pasi, N., \& Kumar, V. (2018). QR Code Based Smart Attendance System. no. October.

[10] Ayop, Z., Yee, C., Anawar, S., Hamid, E., \& Syahrul, M. (2018). Location-aware event attendance system using qr code and gps technology. International Journal of Advanced Computer Science and Applications, 9(9), 466-473.

[11] Cho, D. J., \& Bae, M. Y. (2014). A Study on Development of OTIP System using QR Code based on Smartphone. International Journal of Multimedia and Ubiquitous Engineering, 9(10), 261-270.

[12] Navin, K., Shanthini, A., \& Krishnan, M. M. (2017, August). A mobile based smart attendance system framework for tracking field personals using a novel QR code based technique. In 2017 International Conference On Smart Technologies For Smart Nation (SmartTechCon) (pp. 1540-1543). IEEE.

[13] Khoenkaw, P., \& Pramokchon, P. (2017, March). A software based method for improving accuracy of ultrasonic range finder module. In 2017 International Conference on Digital Arts, Media and Technology (ICDAMT) (pp. 10-13). IEEE.

[14] Erden, F., Alkar, A. Z., \& Cetin, A. E. (2015). A robust system for counting people using an infrared sensor and a camera. Infrared Physics \& Technology, 72, 127-134. 